\title{
Fatigue Life Analysis of One Hybrid Bus Frame Based on Road
}

\section{Spectrum}

\author{
Gaojun Liu, ${ }^{1, a}$, Huaixian Yin ${ }^{2, b}$, Tiezhu Zhang ${ }^{1, c}$, Hongxin Zhang ${ }^{1, d}$, Meishi zhou ${ }^{1, e}$ \\ ${ }^{1}$ Mechanical and Electronic Engineering College, QingDao University, Qingdao 266071, China \\ ${ }^{2}$ Scientific Research Office, QingDao University, Qingdao 266071, China

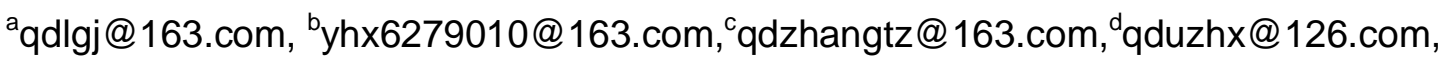

\begin{abstract}
Key words: road spectrum; hybrid bus frame; fatigue analysis
Abstract: To verify the fatigue life of a hybrid bus frame, the finite element model of the frame is built, and the equivalent stress distribution of the frame was got. Multi-body dynamics vehicle model was built in ADAMS/Car. The simulation analysis was conducted based on the road spectrum compiled by MATLAB to simulate $C$ level road model. The load time history of each connection point of the frame was obtained. Combined with the dynamic load analysis and the results of finite element analysis, the fatigue life of the frame is calculated by combing the material S-N curve. Finally, the frame was evaluated comprehensively according to the strength,stiffness, and cumulative fatigue usage. The results could provide a theoretical basis for future optimization.
\end{abstract}

\section{Introduction}

The random vibration of the hybrid bus produced by the excitation of road spectrum will cause fatigue damage of the hybrid bus structure parts, especially the frame which is the main bearing part. The performance of the hybrid bus frame directly affects the safety performance of the whole vehicle, because the frame supports and connects the other parts, and bearing all kinds of loads from inside and outside the vehicle [1]. The fatigue damage is more prominent than other parts, because of its complex structure and uneven force. So the frame's durability test should be carried out in its developing process whose cost is higher and cycle is longer [2]. However, most problem appear after the completion of the product design that bring certain difficulty to the design change. Now today, we can make predictions for the durability of the frame, find the weak link of the structure, put forward reasonable improvement program at the initial stage of product design through the finite element fatigue analysis [3]. What is more, we can also greatly reduce or eventually avoid the fatigue test.

The hybrid bus driving on the $\mathrm{C}$ grade road was simulated throng the multi-body dynamics bus model in this paper, and the dynamic force of the frame bearing is obtained. The finite element model was built and static analysis was carried out with the dynamic force as boundary condition. At last, the fatigue analysis was carried out to verify whether the frame structure is reasonable. The results could provide a base for future optimization.

\section{The establishment of hybrid bus model and road model}

The models of bus suspension, steering system, tire and body model were built with ADAMS/Car in accordance with the data provided by the certain enterprise. The multi-body dynamics bus model is finally obtained through the communication interface of relative assembly. 
The main source of the vibration in the process of the hybrid bus driving was caused by the ground roughness. Therefore, the appropriate road model has a great influence on the accuracy of the simulation. In the main research of road model, there are two ways to get the right road model. The first method is through software to simulate the road and the second is through the actual measured experiment data processing to get the road spectrum.

The road spectrum was obtained by using the first method in this paper. The longitudinal roughness distribution of the $\mathrm{C}$ grade random road was simulated with MATLAB as shown in Figure 1. The road model was built by using Road Builder to compile the road in ADAMS/CAR. Hybrid bus model and road model is shown in Figure 2. The complete hybrid bus model and road model can be simulated.

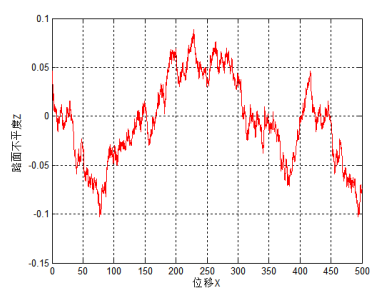

Fig.1 Road spectrum of C grade

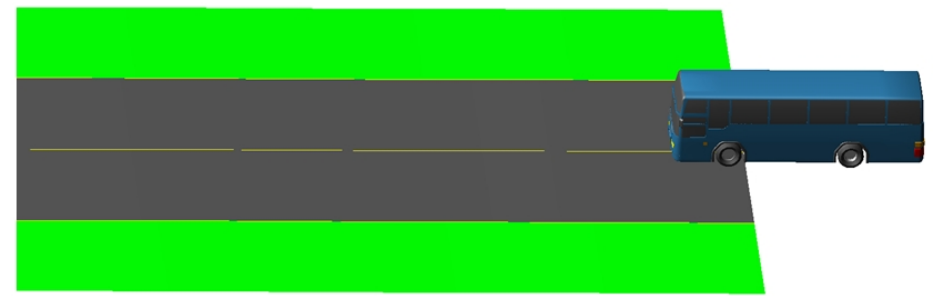

Fig.2 Hybrid bus model and road model

\section{Finite element transient dynamics analysis of the frame}

The simulation method is Straight-Line Event in the Full-Vehicle Analysis. The simulation time takes $15 \mathrm{~s}$. According to its maximum speed, the setting simulation speed is $85 \mathrm{~km} / \mathrm{h}$. After the dynamic analysis, the dynamic force between the frame and the key connection parts were obtained.

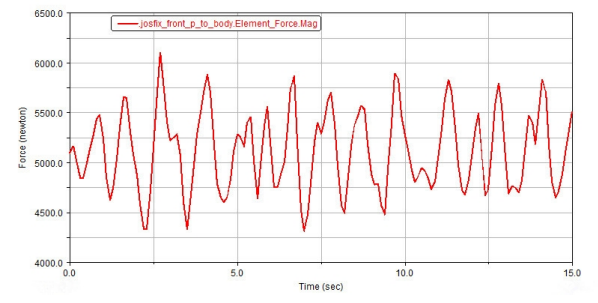

Fig.3 The dynamic force of the front section section

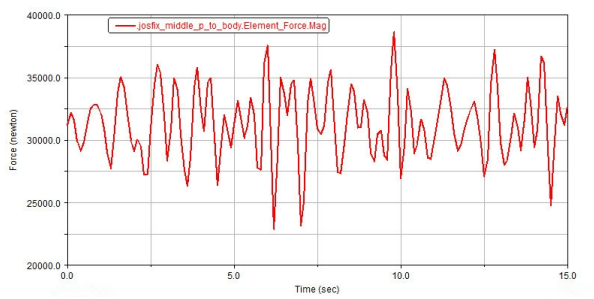

Fig.4 The dynamic force of the middle

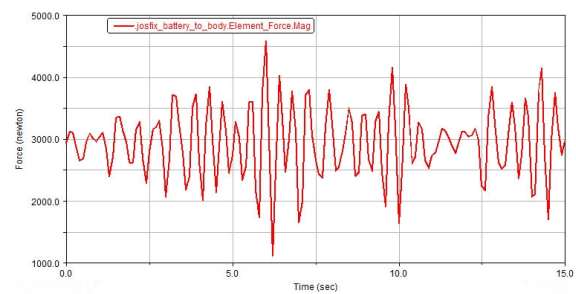

Fig. 6 The dynamic force of battery pack

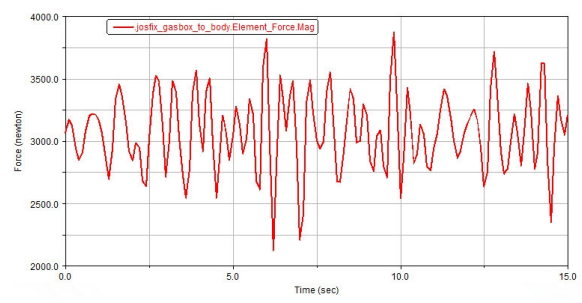

Fig. 8 The dynamic force of natural gas tank
Fig. 7 The dynamic force of power device

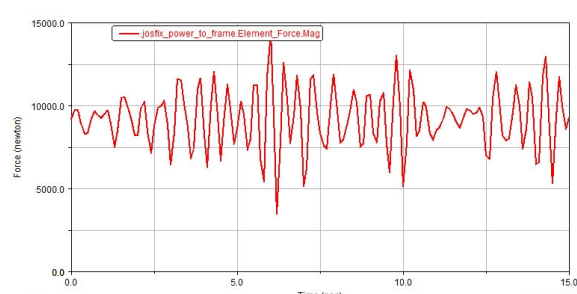

Fig.7 The dynamic force of power device

The dynamic load of the front section hybrid bus frame while hybrid bus is running on random road $\mathrm{C}$ level is shown in Figure 3. The dynamic load of the middle section is shown in Figure 4. The 
dynamic load of the rear section is shown in Figure 5. The dynamic load of the battery pack is shown in Figure 6. The dynamic load of the power device is shown in Figure 7. The dynamic load of the natural gas tank is shown in Figure 8.

\section{Fatigue life analysis of the frame}

Fatigue is a phenomenon of the structure's bearing the repetitive load within the range of a certain load level which is less than the ultimate static load. The fatigue process is that the effect of repeated load leads to internal parts and materials damage and the greatest damage stress level is less than the material yield limit. Therefore, the static strength design can't reflect the actual loading conditions of the frame which is loaded with symmetric fatigue load and alternating cyclic load[4].

There are various ways to obtain the structure stress and strain change, one of the best way is that the transient analysis was carried out with the loads and boundary conditions which vary with time to get the structure stress and strain for every moment. However, this method consumes too much computer resources. The simplified equivalent way was adopt in this paper. We need only obtain the distribution equivalent stress based on the maximum dynamic force in the six key connection parts and the equivalent stress distribution based on the minimum dynamic force. In other words, the dynamic loads were simplified as an alternating cyclic load.

Select the biggest waveform of every key connection point to obtain the maximum dynamic force and the minimum dynamic force. The finite element analysis were carried out with the maximum dynamic force and the equivalent stress distribution is shown in fig.9. The finite element analysis were carried out with the minimum dynamic force and the equivalent stress distribution is shown in fig. 10.

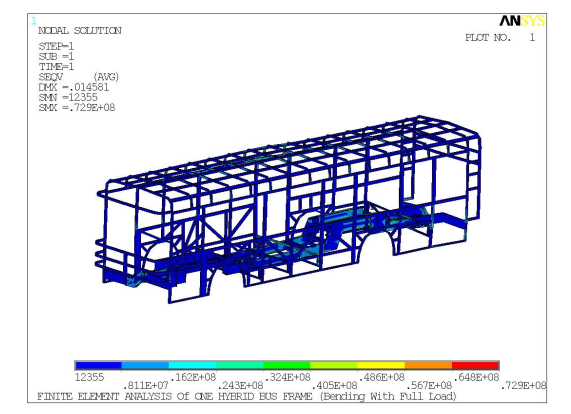

Fig.9 The distribution stress

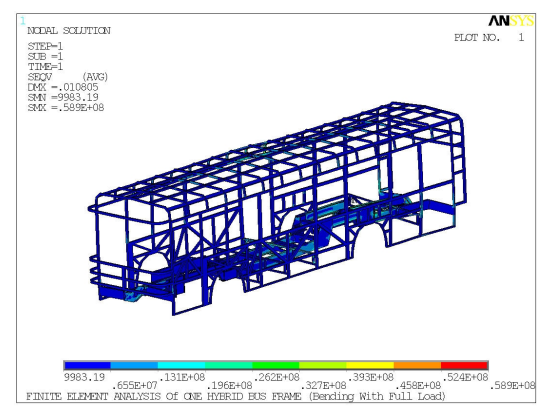

Fig.10 The distribution stress

The maximum equivalent stress with the maximum dynamic force as boundary condition was 72.9 MPa which was far less than the material allowable stress, and the maximum displacement was $0.014 \mathrm{~mm}$, so the strength and stiffness could meet the requirements of the hybrid frame. The maximum stress with the minimum dynamic force as boundary condition was $58.9 \mathrm{MPa}$.

According to the dynamic load analysis and the results of finite element analysis, the fatigue life of the frame is calculated to obtain the specified location of fatigue life by combing the material $\mathrm{S}-\mathrm{N}$ curve. The maximum equivalent stress position of the frame was selected as the specified location to calculate the frame fatigue life in this paper. The fatigue analysis result is shown in fig11. 


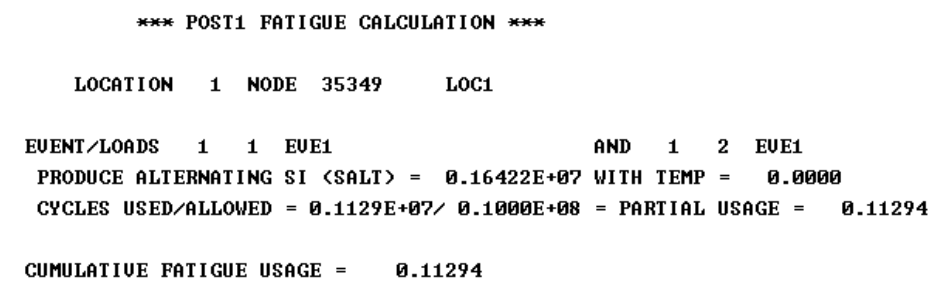

Fig. 11 Fatigue analysis results

The bus should be scrapped when its mileage reached 0.4 million $\mathrm{km}$ according to "Motor vehicle compulsory retirement standards". According to this stipulation, the expected cycle number of the frame calculated is 1.12 million times. The results show that the allowable cycle number of the frame is 10 million times. At this time, the cumulative fatigue usage is 0.11294 that was less than 1. In fact, if the allowable cycle number reached 10 million times, the frame is supposed to have the infinite fatigue life. So the fatigue strength of the frame is surplus, and there is necessary to optimize for the next step work.

\section{Conclusion}

The finite element model of the hybrid bus frame was built, and the equivalent stress distribution of the frame was obtained. The maximum equivalent stress was 72.9 MPa which was far less than the material allowable stress, and the maximum displacement was $0.014 \mathrm{~mm}$, so the strength and stiffness could meet the requirements of the hybrid bus frame. The fatigue life of the frame is calculated. The results show that the allowable cycle number of the frame is 10 million times while the expected cycle number is 1.12 million times. At this time, the cumulative fatigue usage is 0.11294 that was less than 1 . So the hybrid frame design was safe. The final results show that the bus frame can satisfy the usage, and the fatigue strength of the frame is surplus which can provide a theoretical basis for the optimization.

\section{Acknowledgements}

The authors gratefully acknowledge the financial support from the Funded by the National High-tech R\&D Program(863Program) of China(No.2014AA052303), Provincial Shandong Province Foundation of China (No. 2014ZZCX05501 and No. 2014GGX103044).

\section{Reference}

[1] Yang Qingquan, Gu Zhengqi, MI Chengji. Automotive Engineering. 2012,34(11),1016-1019

[2] Huang Minfeng, Jiang Yingchun. Mechanical Research \& Application. 2008,21(2), 57-60.

[3] Gaojun Liu, Tiezhu Zhang, Hongxin Zhang: Applied Mechanics and Materials, Vols. 633-634(2014) pp1148-1153.

[4] Li Nan, Sun Huanwu, Yan Jie. Mechanical Engineering \& Automation. 2012, (5),9-11 